\title{
Prof. Riccardo De Arcangelis
}

\author{
Salvatore Rionero
}

On October 19, 2008, when this issue was about to go to the printers, Prof. Riccardo De Arcangelis, member of the Editorial Board, died suddenly in a tragic accident on the Gran Sasso mountain (at the age of 48). I recall and acknowledge his most helpful contribution to the management of "Ricerche di Matematica", extending over a long time. I am confident that these sentiments also reflect those of all members of the Editorial Board.

Prof. Salvatore Rionero

Editor in Chief 